\title{
Exploring Trade-Offs between Target Coverage, Healthy Tissue Sparing, and the Placement of Catheters in HDR Brachytherapy for Prostate Cancer using a Novel Multi-Objective Model-Based Mixed-Integer Evolutionary Algorithm
}

\author{
Krzysztof L. Sadowski \\ Utrecht University \\ Utrecht, The Netherlands \\ k.l.sadowski@uu.nl \\ Tanja Alderliesten \\ Academic Medical Center \\ Amsterdam, The Netherlands \\ t.alderliesten@amc.uva.nl \\ Yury Niatsetski \\ Elekta \\ Veenendaal, The Netherlands \\ yury.niatsetski@elekta.com
}

\author{
Marjolein C. van der Meer \\ Academic Medical Center \\ Amsterdam, The Netherlands \\ marjolein.vandermeer@amc.uva.nl
}

\author{
Dirk Thierens \\ Utrecht University \\ Utrecht, The Netherlands \\ d.thierens@uu.nl
}

Arjan Bel

Academic Medical Center

Amsterdam, The Netherlands

a.bel@amc.uva.nl

\author{
Ngoc Hoang Luong \\ Centrum Wiskunde \& Informatica \\ Amsterdam, The Netherlands \\ n.h.luong@cwi.nl
}

Rob van der Laarse

Academic Medical Center

Amsterdam, The Netherlands

rob.vanderlaarse@gmail.com

Peter A.N. Bosman

Centrum Wiskunde \& Informatica

Amsterdam, The Netherlands

peter.bosman@cwi.nl

\begin{abstract}
Brachytherapy is a form of radiotherapy whereby a radiation source is guided near tumors, using devices such as catheter implants. In the present clinical workflow, catheters are first placed inside or close to the tumor based on clinical expertise. Subsequently, software is used to design a plan for the delivery of radiation. Treatment planning is essentially a multi-objective optimization problem, where conflicting objectives represent radiation delivered to tumor cells and healthy cells. However, current clinical software collapses this information into a single-objective, constrained optimization problem. Moreover, catheter positioning is typically not included. As a consequence, it is hard to obtain insight into the true nature of the trade-offs between key planning objectives and the placement of catheters. Such insights are however crucial in understanding how better treatment plans may be constructed. To obtain such insights, we interface with real-world clinical software and derive potential catheter positions for real-world patients. Selecting and configuring catheters requires mixed-integer optimization. For this reason, we extend the recently-proposed Genetic Algorithm for Model-Based mixed-Integer opTimization (GAMBIT) to tackle multi-objective optimization problems. Our results indicate that clinically acceptable plans of high quality may be achievable with less catheters than typically used in current clinical practice.
\end{abstract}

Permission to make digital or hard copies of all or part of this work for personal or classroom use is granted without fee provided that copies are not made or distributed for profit or commercial advantage and that copies bear this notice and the full citation on the first page. Copyrights for components of this work owned by others than ACM must be honored. Abstracting with credit is permitted. To copy otherwise, or republish, to post on servers or to redistribute to lists, requires prior specific permission and/or a fee. Request permissions from permissions@acm.org.

GECCO '17, Berlin, Germany

(C) 2017 ACM. 978-1-4503-4920-8/17/07 ..\$15.00

DOI: http://dx.doi.org/10.1145/3071178.3071311

\section{CCS CONCEPTS}

-Mathematics of computing $\rightarrow$ Evolutionary algorithms; Mixed discrete-continuous optimization;

\section{KEYWORDS}

Medicine, Genetic algorithms, Multi-objective optimization, Empirical study

\section{ACM Reference format:}

Krzysztof L. Sadowski, Marjolein C. van der Meer, Ngoc Hoang Luong, Tanja Alderliesten, Dirk Thierens, Rob van der Laarse, Yury Niatsetski, Arjan Bel, and Peter A.N. Bosman. 2017. Exploring Trade-Offs between Target Coverage, Healthy Tissue Sparing, and the Placement of Catheters in HDR Brachytherapy for Prostate Cancer using a Novel Multi-Objective Model-Based Mixed-Integer Evolutionary Algorithm. In Proceedings of GECCO '17, Berlin, Germany, July 15-19, 2017, 8 pages.

DOI: http://dx.doi.org/10.1145/3071178.3071311

\section{INTRODUCTION}

Prostate cancer is the most common type of cancer among men in the Netherlands, a diagnosis that over 10,000 men are confronted with each year, and leading to about 2, 500 deaths per year [11]. Radiotherapy is a commonly applied cancer treatment in which ionizing radiation is used to target tumor cells. An important form of radiotherapy for prostate cancer is brachytherapy (BT), where the tumor is irradiated from inside the body by guiding a radiation source close to or inside the tumor. In this paper, we focus on the case whereby catheters are placed in the prostate, for the radiation source to be moved through. Each catheter contains a number of different positions, called dwell positions, where the source can dwell for prescribed amounts of time, called dwell times. If the source dwells at a given dwell position, that position is called active; 
otherwise, it is called inactive. By varying the dwell times, different treatment plans are possible.

In determining a treatment plan, there are multiple aspects of importance. Firstly, as many tumor cells as possible should obtain a sufficiently high dose in order to maximize the probability of damaging or killing cancer cells. Secondly, the dose to nearby organs cannot be too high, to minimize the probability of complications. Most current clinical software for determining a treatment plan is based on single-objective optimization and manual tuning. However, because of the conflicting nature of these objectives, multi-objective optimization would be a more natural approach since trade-offs can be shown between the different objectives of interest, providing insight into how much may be gained in one objective at what cost to another. This way, a well-informed decision may be taken on what is the best treatment plan for the patient at hand.

Taking also the placement and number of catheters into account in the multi-objective optimization perspective is a natural fit, because the exact location of catheters governs much of what may be achieved in terms of plan quality. Moreover, to minimize the possibility of complications, one should use as few catheters as possible $[6,8,14]$. Currently, the placement of catheters is done mostly based on medical expertise. At the hospital involved in this study there are typically $16-18$ catheters used. Many placements are possible and for each placement many treatment plans are possible, comprising a vast search space. Even though expertise and literature exists on how to place catheters, there is little information on the real-world trade-offs of catheter placements. Our goal is to take a first step in this direction, obtaining such insights, learning about the complexity of the multi-objective optimization problem, and start to work toward algorithms that may really be used for patient-specific optimization in clinical practice. The novelty in our approach is bringing together several aspects that were not considered altogether before. Multi-objective approaches to treatment planning have been proposed, including catheter placement via the weighted-sum method in combination with single-objective optimization, but did not optimize directly on the evaluation criteria of a clinical evaluation protocol [9]. Related work that does optimize on these criteria exists, but is single-objective and does not consider catheter placement [5]. In contrast, we consider the use of realworld clinical software and patients combined with multi-objective optimization directly on criteria as considered in clinical evaluation protocols.

From an algorithmic perspective, Evolutionary Algorithms (EAs) are among the state-of-the-art when it comes to solving multiobjective optimization problems [4]. For this reason, we are interested in using an EA to solve this problem. The problem includes both discrete (which catheters) and continuous (dwell times) variables. Many state-of-the-art (multi-objective) EAs focus on either discrete or continuous domains exclusively. Optimization where discrete and continuous variables are present simultaneously is explored relatively less and is referred to as mixed-integer optimization. A recently introduced Genetic Algorithm for Model-Based mixed-Integer opTimization (GAMBIT) has shown to be an effective approach to single-objective optimization in the mixed-integer domain [12], especially in the case of black-box optimization, meaning that no internal structure of the problem is assumed to be known in advance. This makes GAMBIT flexible and easily adaptable for our multi-objective approach of BT pre-planning optimization.

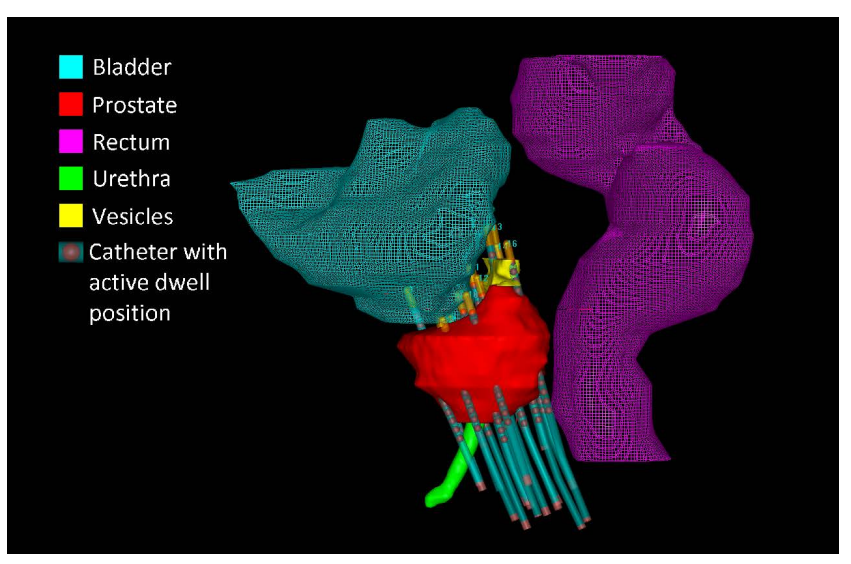

Figure 1: Graphical representation of a patient's anatomy and implanted catheters.

\section{BT TREATMENT PLANNING}

\subsection{Clinical practice}

In BT for prostate cancer, the treatment targets are the prostate and the seminal vesicles (see Fig. 1). The Organs At Risk (OARs), which should be radiated as little as possible, are the nearby organs, i.e., bladder, rectum, and urethra. Guided by live ultrasound images, a typical High-Dose-Rate (HDR) prostate BT treatment starts with implanting a number of catheters into the prostate through the patient's perineum skin (between scrotum and anus). The suitable number of catheters and their proper positions depend on the size of the prostate and its geometry in relation to other nearby organs (e.g., bladder and rectum). The inserted catheters need to be firmly fixed to prevent displacements. The patient is subsequently transferred to the imaging room, where computed tomography (CT) or Magnetic Resonance Imaging (MRI) scans of the pelvic cavity are acquired. These scans are then used to finalize the treatment plan.

First, the catheters, the targets, and OARs are delineated on the CT/MRI scans in planning software. The doctors then specify which dwell positions in each catheter should be activated. For a given dwell time of a source at a dwell position, more radiation dose is delivered to nearby tissues than to faraway tissues. Therefore, to increase the probability of fully treating the target and sparing healthy tissues at the same time, dwell positions inside the target volumes are activated while the ones far away from the targets (e.g., at distances larger than $5 \mathrm{~mm}$ ) or too close to OARs are normally not considered. A trivial treatment plan, in which dwell times take on very large values, can kill all tumor cells but also causes necrosis (i.e., non-physiological cell death) to healthy tissues. On the other hand, another trivial treatment plan, where all dwell times are zero, can effectively spare healthy tissues from radiation risks but such a plan equals no treatment at all.

A specific setting of dwell times delivers a certain radiation dose distribution to the tissues surrounding the catheters. The dose distribution needs to be assessed by doctors to determine 
if such a setting can be clinically acceptable. A radiation dose is prescribed for the entire prostate, termed the prescribed dose. Tumor cells, which are more susceptible to radiation than healthy cells, should receive at least the prescribed dose to be effectively killed. Also, while healthy cells could recover from being radiated at the prescribed dose level, they should not be radiated too much; otherwise, healthy cells would be killed as well. However, it is impossible to compute the exact amount of radiation received by every cell since the number of cells is prohibitively numerous. Dose distributions, therefore, are often approximated by only computing the radiation dose at a certain number of so-called dose calculation points. Of key interest is how large the cumulative volume of an organ covered by a certain dose is. Such information, termed DoseVolume-Histogram (DVH) indices, can be represented as $V_{x}^{o}$ : the volume (expressed either relatively or absolutely) of organ $o$ that received at least radiation dose level $x$. For example, international GEC/ESTRO recommendations [7] state that treatment plans should result in $V_{100}^{\text {prostate }} \geq 95 \%$, i.e., the prostate volume that receives at least $100 \%$ of the prescribed dose should be $\geq 95 \%$ of the total prostate volume. To prevent necrosis, it is required that $V_{200}^{\text {prostate }} \leq$ $20 \%$, i.e., the prostate volume covered by $200 \%$ of the prescribed dose should $\leq 20 \%$ of the total prostate volume. $V_{100}^{\text {prostate }}$, therefore, is a target coverage index while $V_{200}^{\text {prostate }}$ is an organ sparing index. Similarly, there exist other sparing DVH indices for other OARs. For example, it is recommended that $V_{110}^{\text {urethra }} \leq 0.1 c c$, i.e., the urethra volume covered by $110 \%$ of the prescribed dose should be $\leq 0.1 c c$. Finally, because the vesicles may also contain tumor cells, there is a constraint that $V_{80}^{\text {vesicles }} \geq 95 \%$. A full description of the current clinical protocol in terms of DVH indices at the hospital involved in this study is presented in Table 1.

\begin{tabular}{|c|c|c|c|c|}
\hline Prostate & Bladder & Rectum & Urethra & Vesicles \\
\hline$V_{100} \geq 95 \%$ & $V_{86} \leq 1 c c$ & $V_{78} \leq 1 c c$ & $V_{110} \leq 0.1 c c$ & $V_{80} \geq 95 \%$ \\
\hline$V_{150} \leq 50 \%$ & $V_{74} \leq 2 c c$ & $V_{74} \leq 2 c c$ & & \\
\hline$V_{200} \leq 20 \%$ & & & & \\
\hline
\end{tabular}

Table 1: BT treatment planning in clinical practice at the involved hospital: DVH index criteria.

The values of DVH indices in the clinical protocol are consulted by the doctors when making treatment plans. Planning often starts with an initial plan proposed by BT treatment planning software. Note that it is difficult, however, to directly optimize DVH indices due to their discrete nature. For example, maximizing the $V_{100}^{\text {prostate }}$ index equals maximizing the number of dose calculation points inside the prostate that receives at least the prescribed dose. To quickly achieve a result, planning software therefore often solves simplified optimization models of the problem with local search methods. It is thus not guaranteed that a treatment plan proposed by available software satisfies all DVH index criteria in the protocol or that the plan is the best possible one for the patient case at hand. Medical planners then need to manually adjust this proposed plan. They often first consider the DVH index that is violated the most compared to the recommended protocol, and try to find the locations of violation, then change the dwell times of nearby dwell positions to improve the index until satisfied. They then continue with a different DVH index that is now the most violated. Note that improving a DVH index might deteriorate other indices, including ones that have been previously worked on. The plan is adjusted in such an iterative manner until the doctors are satisfied.

How good the DVH indices can be, depends on the quality of the catheter implant and the geometry of the involved organs. Certain implants will not allow all DVH indices to satisfy the recommended protocol. For example, with too few or improperly placed catheters, it is difficult to obtain $V_{100}^{\text {prostate }} \geq 95 \%$ while meeting constraints on indices of OARs. Similarly, if the rectum is too close to the prostate, $V_{78}^{\text {rectum }} \leq 1 c c$ and $V_{100}^{\text {prostate }} \geq 95 \%$ might not be achievable at the same time. In such situations, for each specific case, the doctors need to decide which indices are more important to be satisfied and which indices can be compromised. Final approved treatment plans, thus, might (slightly) violate some clinical protocol thresholds.

\subsection{Multi-Objective Treatment Planning}

BT treatment planning is intrinsically a multi-objective optimization problem. The DVH indices in Table 1 can be categorized into two groups: treatment target indices (i.e., $V_{100}^{\text {prostate }}$ and $V_{80}^{\text {vesicles }}$ ) and organ sparing indices. Candidate treatment plans that do not achieve the minimum requirements on the indices in the clinical protocol are less favorable than candidate plans that satisfy all protocol thresholds. For a treatment target index, the larger its value is (above the corresponding threshold), the better it is. For an organ sparing index, the lower its value is (below the corresponding threshold), the better it is. Optimizing treatment target indices results in increasing dwell times to make the target volumes covered by the treatment dose level as large as possible, where the best value is $100 \%$. On the other hand, optimizing organ sparing indices results in decreasing dwell times to make the organ volumes covered by the radiation risk dose levels as small as possible, where the best value is $0 \% / 0 c c$. The two groups of DVH indices conflict with each other such that a utopian treatment plan yielding $100 \%$ for all target coverage indices and $0 \% / 0 c c$ for all organ sparing indices does not exist. Instead, the optimum of this multi-objective problem is a set (i.e., Pareto-optimal set) of equally-good alternative plans which are optimal in the sense that improving one objective deteriorates the other objective, and vice versa.

There are nine different DVH index criteria. We argue, however, that the clinical protocol can be reformulated to a bi-objective optimization problem without losing key insight into trade-offs by combining the DVH indices of the same group (i.e., target coverage or organ sparing) into a representative objective of that group. To this end, we employ a risk-averse perspective, meaning that a treatment plan is evaluated to be only as good as the worst target coverage index and the worst organ sparing index. More specifically, each candidate plan has two objective values: the Least Coverage Index (LCI), which corresponds to the worst-scored DVH index in the target coverage group, and the Least Safe Index (LSI), which corresponds to the worst-scored DVH index value in the organ sparing group. Therefore, for a specific treatment plan, all indices in a group are at least as good as the representative value of the group. Such an approach has an analogy with the clinical practice in the iterative focus on improving the current worst index at each time as mentioned previously. Moreover, unlike the weighted-sum 
approach, where all criteria are added together, each DVH index in our problem modeling is still, in effect, considered separately.

The feasible search space is enlarged to contain solutions that violate the clinical protocol to some degree. There are two reasons for this. The first is that, in this way, the clinically-feasible solutions can be approached from both the clinically-feasible and the clinically-infeasible space, making the search in these regions more efficient. Secondly, we account for the fact that sometimes there exists no treatment plan satisfying all clinical protocols (due to the quality of the implants and/or the geometry of the involved organs, see Section 2.1), and a treatment plan that (slightly) violates the protocol must thus be accepted. To relax the clinical constraints, the new upper bounds for organ sparing indices are increased four times (e.g., $V_{86}^{\text {bladder }} \leq 1 c c$ in the protocol becomes $V_{86}^{\text {bladder }} \leq 4 c c$ in the optimization model). The new lower bounds for target coverage indices are also decreased accordingly as: $V_{100}^{\text {prostate }} \geq 80 \%$ and $V_{80}^{\text {vesicles }} \geq 80 \%$. The optimization constraints are presented in Table 2. Let $V_{x}^{\max }$ denote the clinical protocol threshold of a dose level $x$ for an organ. To measure and normalize the distance of an organ sparing index value to its corresponding upper bound, we define $\delta\left(V_{x}\right)=1-\frac{V_{x}}{4 \times V_{x}^{\max }}$. The larger the value of $\delta$ is, the better the corresponding DVH index value $V_{x}$ of that organ is and how safe an organ is compared to the relaxed protocol threshold, with a value of 0.75 meaning that the clinical protocol constraint is met. The LSI objective value is defined as the minimum $\delta$ value among all organ sparing indices. Similarly, the LCI objective value of a treatment plan is defined as the minimum coverage value among all target coverage indices. For example, a treatment plan with $V_{100}^{\text {prostate }}=97 \%, V_{80}^{\text {vesicles }}=96 \%, V_{150}^{\text {prostate }}=19 \%, V_{200}^{\text {prostate }}=$ $7 \%, V_{86}^{\text {bladder }}=0.5 c c, V_{74}^{\text {bladder }}=1.3 c c, V_{78}^{\text {rectum }}=0.4 c c, V_{74}^{\text {rectum }}=1.2 c c$, $V_{110}^{\text {urethra }}=0.1 c c$ would have the Least Coverage Index value as 0.96 (taking the value of $V_{80}^{\text {vesicles }}$ ) and the Least Safe Index value as 0.75 (corresponding with $V_{110}^{\text {urethra }}$ ).

\begin{tabular}{|c|c|c|c|c|}
\hline Prostate & Bladder & Rectum & Urethra & Vesicles \\
\hline$V_{100} \geq 80 \%$ & $V_{86} \leq 4 c c$ & $V_{78} \leq 4 c c$ & $V_{110} \leq 0.4 c c$ & $V_{80} \geq 80 \%$ \\
\hline$V_{150}$ free & $V_{74} \leq 8 c c$ & $V_{74} \leq 8 c c$ & & \\
\hline$V_{200} \leq 80 \%$ & & & & \\
\hline
\end{tabular}

$$
\begin{aligned}
& f_{1}= \min \left\{V_{100}^{\text {prostate }}, V_{80}^{\text {vesicles }}\right\} \\
& f_{2}=\min \left\{\delta\left(V_{150}^{\text {prostate }}\right), \delta\left(V_{200}^{\text {prostate }}\right), \delta\left(V_{86}^{\text {bladder }}\right), \delta\left(V_{74}^{\text {bladder }}\right), \delta\left(V_{78}^{\text {rectum }}\right),\right. \\
&\left.\quad \delta\left(V_{74}^{\text {rectum }}\right), \delta\left(V_{110}^{\text {urethra }}\right)\right\}
\end{aligned}
$$

$f_{3}=$ number of catheters used.

Table 2: Multi-objective optimization model for BT treatment planning. $f_{1}$ and $f_{2}$ should be maximized while $f_{3}$ should be minimized. Because of the relaxation factor, $V_{150}^{\text {prostate }}$ has become unconstrained.

In this paper, we focus on the pre-planning phase, i.e., before the catheters are inserted. To generate data and problem input that corresponds to real-world clinical practice, we are currently bound to real-world clinical software. Given a prostate BT patient, a number of possible catheter configurations can be determined, after which the clinical software provides information on these possible catheter configurations and their corresponding dwell positions.
Hence, there is a restriction in the sense that the optimization can only use pre-determined catheter positions. In the software many catheters can be placed, but in a real patient it is desirable to have catheters not too close to each other to avoid complications. In order to have both a large enough search space for interesting first results and a set of configurations in which catheters are not too close to each other, we chose the set of possible configurations to consist of 30 catheters for each patient.

The optimization problem now contains $d$ discrete and $c$ continuous variables. The $d$ discrete variables represent all the possible catheter placement positions. Each of these variables takes on a binary value, where 1 represents placing the corresponding catheter, and 0 not placing the catheter. Each of the continuous variables represents the dwell time corresponding to a dwell position in a catheter. Dwell times for dwell positions inside an inactive catheter can be ignored.

\section{MODEL-BASED MULTI-OBJECTIVE MIXED-INTEGER EVOLUTIONARY ALGORITHM}

In this section we summarize key concepts and terminology pertaining to mixed-integer and multi-objective optimization and provide an overview and summary of GAMBIT, and introduce the MultiObjective GAMBIT (MO-GAMBIT).

\subsection{Terminology}

3.1.1 Mixed-integer optimization. A mixed-integer problem is defined as follows:

$$
\begin{gathered}
\max f\left(x_{d}, x_{c}\right) \\
\text { s.t. } \mathbf{h}\left(x_{d}, x_{c}\right)=0, \mathbf{g}\left(x_{d}, x_{c}\right) \leq 0
\end{gathered}
$$

Here, $x$ represents the solution

$$
x=x_{d} x_{c}=d_{0} \ldots d_{l_{d}-1} c_{0} \ldots c_{l_{c}-1}
$$

where $d_{i} \in\{0,1\}, c_{i} \in \mathbb{R}$, and $x_{d}, x_{c}$ are the groups of all discrete and real-valued variables, respectively. $l_{d}$ and $l_{c}$ represent the number of discrete and continuous variables. Moreover, $f$ is the objective function, and $\mathbf{h}$ and $\mathbf{g}$ are the sets of equality and inequality constraint functions, respectively. If both sets are empty, the mixed-integer problem is said to be unconstrained.

3.1.2 Multi-Objective Optimization. A Multi-Objective (MO) optimization problem consists of $m$ objectives $f_{i}(\boldsymbol{x}), i \in\{0,1, \ldots, m-$ $1\}$, that without loss of generality, must all be maximized. The objective value vector of a solution $\boldsymbol{x}$ is $\boldsymbol{f}(\boldsymbol{x})=\left(f_{0}(\boldsymbol{x}), f_{1}(\boldsymbol{x}), . ., f_{m-1}(\boldsymbol{x})\right)$. A solution $x^{0}$ dominates a solution $x^{1}$ (denoted $x^{0}>x^{1}$ ) if and only if $f_{i}\left(x^{0}\right) \geq f_{i}\left(x^{1}\right), \forall i \in\{0,1, \ldots, m-1\}$ and $f\left(x^{0}\right) \neq f\left(x^{1}\right)$. A solution $x^{0}$ is Pareto optimal if and only if there does not exist a solution $\boldsymbol{x}^{1}$ such that $\boldsymbol{x}^{1}>\boldsymbol{x}^{0}$. The Pareto-optimal set $\mathcal{P}_{S}$ of the problem at hand is the set of all Pareto-optimal solutions. The Pareto-optimal front $\mathcal{P}_{\boldsymbol{F}}$ is the set of the objective value vectors of all Pareto-optimal solutions. The goal of MO optimization is to find a set of non-dominated solutions whose objective value vectors constitute a good approximation of the Pareto-optimal front [4]. 


\subsection{GAMBIT}

GAMBIT is a recently introduced EA aimed at solving mixed-integer problems, especially in the black-box setting. Here, we give a brief outline of GAMBIT. More details can be found in literature [12]. GAMBIT is a parameter-free model-based EA capable of learning and exploiting different types of variable dependencies, through a clustering mechanism and an integrated dependency-models mechanism. The ability to learn such dependencies (i.e., which groups of variables have a joint synergistic effect on a solution's quality) allows for the creation of variable subsets which represent important building blocks of the problem. These building blocks can be used to exploit problem structure and generate new and better solutions more effectively. Such exploitation of problem structure based on dependencies is well-established for discrete and continuous variables, respectively. Mixed-integer optimization introduces new optimization challenges, however. The existence of discrete and continuous variables creates a potential for intra- and intervariable dependencies that domain-specific model-based EAs are not equipped to handle. GAMBIT therefore introduces a new way to learn and process mixed inter-domain variable dependencies, alongside intra-domain dependencies (i.e., in individual domains) during optimization.

GAMBIT splits the population in each generation with the use of a clustering algorithm as detailed in Figure 2. Each of the subpopulations acquired is then subject to variation through the integrated dependency models mechanism, summarized in pseudo-code in Figure 3. Specifically, a Family-Of-Subsets (FOS) model is learned at the beginning of every generation, which is used to describe dependencies and to create offspring solutions [13]. Essentially, a FOS is a set of linkage sets. Each linkage set contains the indices of the variables that are considered to be dependent. The linkage sets may overlap. The FOS consists of three types of subsets: discrete, continuous, and mixed. In its full black-box optimization configuration discrete subsets are generated using a so-called linkage tree, which is a specific type of FOS that was first used in the algorithm known as Linkage Tree Genetic Algorithm (LTGA), which is now considered to be an instance of the Gene-pool Optimal Mixing Evolutionary Algorithm (GOMEA) family [13]. The linkage tree is a FOS structure acquired by building from the bottom up (i.e., starting with singleton subsets of problem variables) a tree of subsets by means of a hierarchical clustering algorithm based on mutual information between pairs of variables. The linkage tree has $2 l_{d}-1$ discrete subsets representing important building blocks in the discrete domain. The same number of continuous subsets are added to the FOS, each containing every continuous problem variable. Additionally, $l_{c}+l_{d}$ mixed subsets are added to the FOS by building another linkage tree constrained to merge discrete and continuous variables using a mixed mutual information metric, described in detail in [12]. Such mixed subsets allow for the consideration of discrete and continuous variables together, resulting in the ability to exploit potential mixed variable dependencies. Each type of subset is processed with a corresponding mechanism type to generate new solutions. Once all subsets are processed, new solutions replace the previous population and the clustering process begins anew.

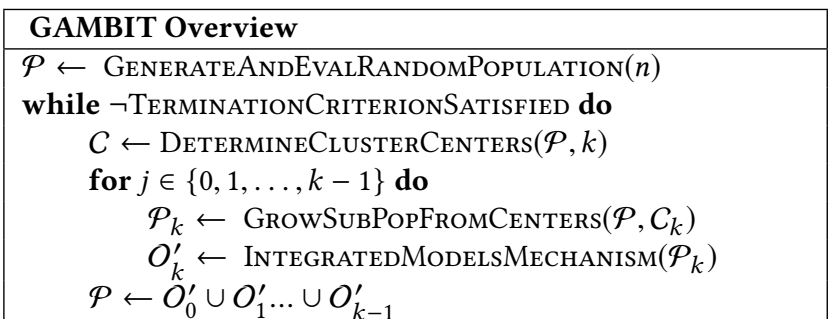

Figure 2: Pseudo-code overview of GAMBIT. $\mathcal{P}$ represents the population of size $n$. $C$ contains $k$ cluster (also called subpopulation) centers. $\mathcal{P}_{i}$ represents the $i$-th sub-population and $O_{i}^{\prime}$ is the offspring generated from sub-population $\mathcal{P}_{i}$.

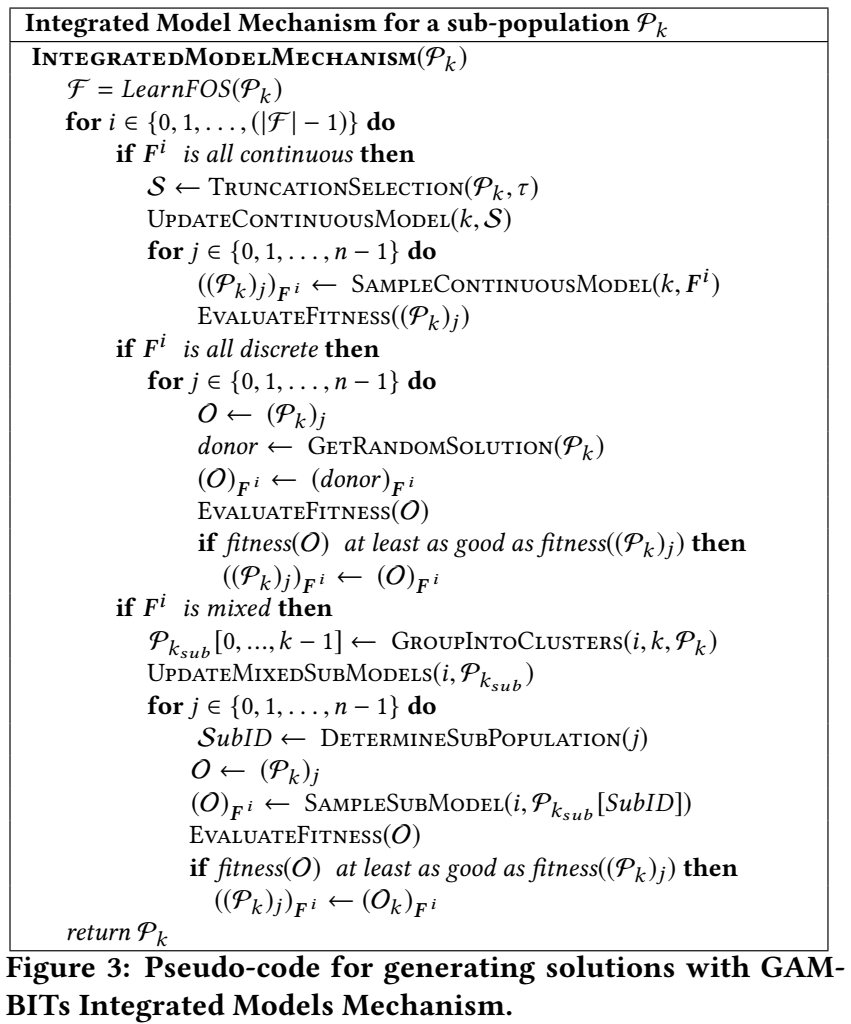

\subsection{Multi-Objective GAMBIT}

In this paper we introduce MO-GAMBIT that brings the blackbox mixed-integer optimization capabilities of GAMBIT into the MO domain. The approach to handle MO problem landscapes with GAMBIT is to include mechanisms designated specifically to exploit the multi-objective nature of the problem landscape, such as an elitist archive and the ability to rank solutions in a multi-objective space. In this section, we outline the key components used in the design of MO-GAMBIT and its specific application to brachytherapy pre-treatment planning.

3.3.1 Elitist Archive. In previous work on multi-objective EAs, elitism has been shown to be very important [3]. For this reason, an elitist archive is added in MO-GAMBIT. Because real-valued variables are involved, we use a technique that adaptively changes 
the grid that governs the elitist archive so as to harbor a predefined maximum number of solutions, preventing occurrences of very similar solutions in the archive while promoting diversity. For more details, see [10].

3.3.2 Selection and Variation. The selection and variation mechanisms are changed in MO-GAMBIT to account for the MO nature of the problems, and to make use of the elitist archive. Solutions are still clustered at every generation, however clustering is performed in the objective space. This allows MO-GAMBIT to specialize model-based optimization in different areas of the Pareto front. Variation proceeds by improving existing solutions, as is reminiscent of GOMEA, in case of discrete and mixed linkage sets. Solution parts are copied from donors and mixed into solutions, and changes are only accepted in case of a multi-objective improvement. Continuous variables are supplied from normal distributions estimated from a population-based MO rank-based selection [3] following procedures of the real-valued EDA known as iAMaLGaM [2] and its multi-objective counterpart iMAMaLGaM [1]. For an overview in pseudo-code, see Figure 3.

Upon sampling new solutions, the clusters are populated with solutions from the previous generation as well as a fraction of $\tau=0.35$ elitist archive solutions that are closest to the given cluster in the objective space, using a Euclidean distance metric. If not enough elitist archive solutions exist, the entire archive is used for each cluster. This combination of solutions from the previous generation and the elitist archive is used to estimate and sample new solutions. Previously, in MO versions of GOMEA and iAMaLGaM this was found to have a positive effect on convergence to highquality solutions $[1,10]$.

3.3.3 Population and Cluster Sizing. Determining parameters such as the population size or number of clusters can be crucial for the effectiveness of EAs. Small population sizes or insufficient number of clusters can lead to premature convergence. Conversely, too many clusters or too large population sizes can result in a very large overhead. To avoid the need to specify these parameters, MO-GAMBIT adapts a interleaved multistart scheme, which dynamically introduces larger population sizes. Specifically, the scheme runs differently sized instances of GAMBIT in an interleaved fashion. For every $\gamma=2$ generations of a certain instance of GAMBIT, one generation of an instance of GAMBIT with twice the population size and one more cluster is performed. By doing so, smaller population sizes perform more generational steps than larger ones. The scheme already used with GAMBIT is altered to make use of the elitist archive. With GAMBIT, all the differently sized instances are completely independent of each other. In MOGAMBIT the elitist archive is shared over all instances, allowing to transfer knowledge about high-quality solutions already obtained with the smaller populations.

3.3.4 Problem-Specific Knowledge. A strictly black-box optimization algorithm may be a great starting point when considering new problems. In a real-world setting however, some problemspecific information can potentially improve performance. In our case consider that every discrete variable $d_{0}, d_{1}, \ldots, d_{l_{d}-1}$ is associated with an independent set of dwell positions. Instead of learning mixed-variable dependencies, which could result in a significant overhead, a pre-defined mixed-variable subset structure is defined that captures key dependencies. Specifically, each subset contains one discrete variable (representing the catheter) and the set of continuous variables that represent the dwell positions associated with this catheter. Moreover, because we do not expect strong dependencies to exist between continuous variables, we do not model them jointly in one linkage set as per default in GAMBIT, but rather model them independently, i.e., univariately to reduce substantially the overhead of sampling high-dimensional Gaussian distributions.

3.3.5 Sliced 3-objective optimization. We minimize the number of catheters in one of the objectives. However, we slice this 3-dimensional problem into many 2-dimensional multi-objective problems. Little relation between configurations with different numbers of catheters exists, and switching a catheter on (i.e., changing a binary variable from 0 to 1 ) has huge impact, implying there exist big jumps in the search space across different numbers of catheters, making a direct 3-objective optimization approach extra difficult. A sliced approach allows an instance of GAMBIT to focus on configurations with the same number of catheters, while at the same time making it easier to parallelize the optimization across configurations with different number of catheters. We refer to our approach as a multi-layer approach, where in a given layer only combinations with the of number of catheters are allowed. Because solutions resulting in a different numbers of catheters can be created in variation, a simple random repair mechanism is used to activate or deactivate a required number of catheters.

\section{EXPERIMENTS}

\subsection{Setup}

Clinical data from three recently treated patients is used for optimization. For each patient, there are 30 possible catheter locations, resulting in $30 \mathrm{MO}-\mathrm{GAMBIT}$ instances, each performing optimization on a 2-objective mixed-integer problem. The duration of optimization for each MO-GAMBIT instance is limited to 48 hours (on an AMD Opteron(tm) Processor 6386 SE and Intel(R) Xeon(R) CPU E5 2699 v4), with all 30 configurations executed in parallel. During the optimization 20,000 randomly chosen dose calculation points are used to compute the objective values for every function evaluation. To ensure sufficient accuracy in reporting final outcomes, the final set of solutions on the Pareto front is re-evaluated with 100,000 dose calculation points, which is commonly considered to be sufficiently accurate.

\subsection{Results}

Results in Figure 4 illustrate the quality of solutions which can be acquired with different numbers of catheters. The results provide a clear illustration of the trade-offs between the objectives. A solution that satisfies clinical protocol lies in the area of the Pareto front where $\mathrm{LCI} \geq 0.95$ while LSI $\geq 0.75$. The clinically approved solutions for the considered patients all used 16 catheters. Figure 4 shows that it is possible to obtain approvable, high-quality plans using smaller numbers of catheters. Very little quality of solutions is lost when using a few less catheters. Only when many less catheters are used (less than 9 for the tested patients) the resulting solutions 

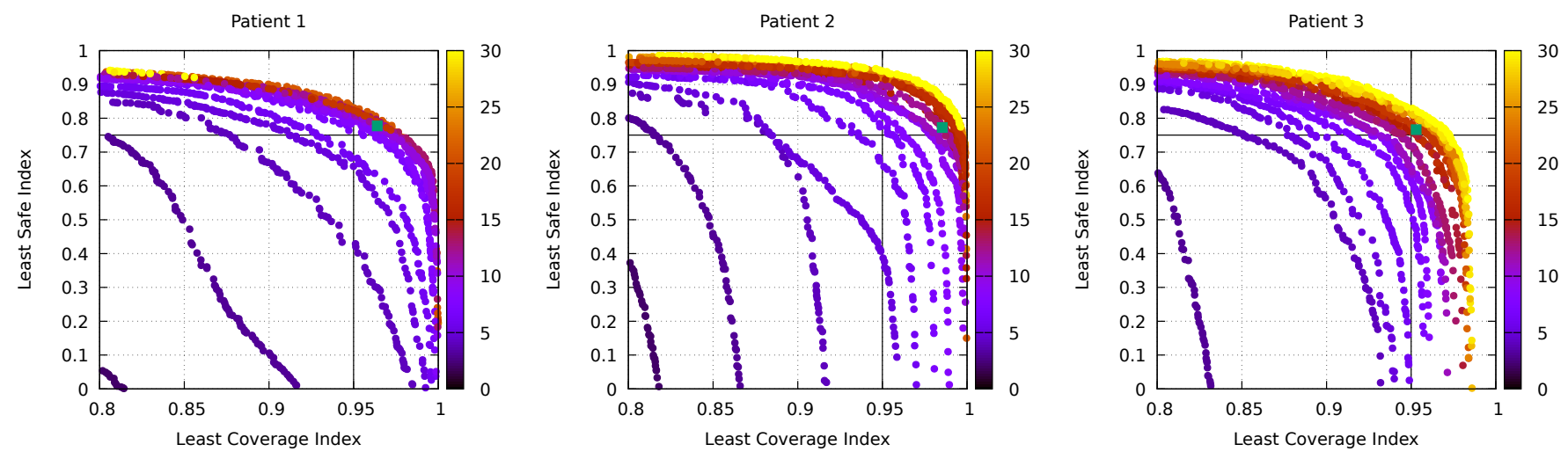

Figure 4: Pareto fronts obtained with MO-GAMBIT illustrating trade-offs between three objectives for three patients. The color coding represents the number of catheters. Green points represent the high-quality solutions selected for visualization in Figure 5 with 10,12 and 16 catheters for patients 1,2 and 3 respectively.

lead to clearly inferior and unacceptable plans when considering the other two objectives.

Outcomes still differ widely between patients. More solutions with better objective values are found for patient 2 . Increasing the number of catheters for patient 2 provides bigger benefits in terms of the LCI and LSI objectives than for patient 1. This suggests that plan optimization can substantially differ for different patients, further highlighting the potential benefits of enabling insight into possible trade-offs and especially the added value of considering the number of catheters as part of optimization.

When the number of catheters is small, the addition of catheters can substantially improve the trade-offs between target coverage and organ sparing (e.g., see the gap between the 2D Pareto fronts of using less vs more than 5 catheters). However, when the number of catheters is sufficient, the trade-off improvement due to adding more catheters clearly diminishes. It would be interesting for future work to investigate the marginal added value of each catheter insertion with respect to the (2D) Pareto-front improvement when considering a larger patient group.

The severity of trade-offs between the LCI and LSI is much higher in the more extreme regions of the Pareto front. For example, at the area where LCI is larger than 0.95 , trying to increase target coverage can quickly worsen the sparing of OARs. On the other hand, if a treatment plan of LCI $=0.95$ is satisfiable, the healthy tissues can be considerably spared from radiation risks (e.g., compare the high value of LSI of solutions having LCI $=0.95$ ). This is very likely part of the notorious practical hardness of deciding upon what values for a good plan to ultimately use.

Figure 5 illustrates selected plans obtained with MO-GAMBIT with only 10,12 and 16 catheters for patients 1, 2 and 3 respectively. All DVH indices satisfy the clinical constraints. For these plans a doctor can decide whether it is acceptable, or whether a factor should be improved at the expense of another factor. For example, it may be desirable to use less than 12 catheters for patient 2, even with worse target coverage (but still satisfying all constraints). From the complete set of Pareto optimal solutions, a candidate plan in that trade-off direction can be taken, for which the same decision can be made, until finally the best Pareto-optimal solution is chosen without optimization needing to be re-ran.

\section{DISCUSSION AND CONCLUSIONS}

This paper considers BT treatment pre-planning optimization from a multi-objective perspective, while combining, for the first time, a true multi-objective optimization approach with optimization directly on clinical evaluation criteria and considering trade-offs between key objectives: the covering of targets, the sparing of organs at risk, and the number of catheters. In current medical practice devising such plans depends heavily on the expertise of doctors. We wish to assist them by enabling the ability to view and consider a set of approximated Pareto optimal solutions with respect to the aforementioned objectives. With this ability, doctors may gain additional insight into possible treatment plans, potentially resulting in improved patient care. As a first proof of concept to this end, we combined real-world clinical software with a novel extension of GAMBIT to multi-objective optimization.

The generated three-objective Pareto fronts for real-world patients provided a useful overview of possible treatment plans with respect to the number of catheters used, potential risk to healthy organs, and the coverage of the targets. Including, for the first time, the number and location of possible catheters placements with a multi-objective optimization approach allowed us to gain new, clinically relevant insight into BT pre-planning optimization. We were able to obtain sets of clinically acceptable non-dominated solutions, and indicated that it appears possible to obtain good solutions with smaller numbers of catheters compared to the clinically used plans for the tested patients. Using a larger number of catheters can lead to better, clinically acceptable solutions in terms of the other objectives, but the added value in key DVH indices used to evaluate plans strongly diminishes when using more than around 10 catheters. Outcomes further present insights into tradeoffs between other key objectives, allowing doctors to utilize their expertise, and consider which of such trade-offs may be beneficial to the patient without re-running optimization.

Our approach based on MO-GAMBIT provides a new, promising and insightful approach for optimization of BT treatment plans, 
but further improvements to this approach are needed and possible. Optimization mechanisms which consider problem structure specifics such as evaluation decomposability could potentially improve optimization speed substantially. Combined with other problem-specific mechanisms, such as a multi-resolution approach to increasing the accuracy of dose calculations over time, a manyfold speedup may be obtained, reducing required run times to minutes rather than hours (even though in the pre-planning phase this is far less of an issue than when making treatment plans after physical catheter placement has taken place) and allowing for more exhaustive exploration of the search space, potentially leading to discovery of even more high-quality non-dominated solutions.

\section{ACKNOWLEDGEMENTS}

This work is part of the research programmes Vrije Competitie (project number 612.001.111) and IPPSI-TA (project number 628.006.003) which are financed by the Netherlands Organisation for Scientific Research (NWO). The project in the IPPSI-TA programme is cofinanced by Elekta.

\section{REFERENCES}

[1] P.A.N. Bosman and T. Alderliesten. 2012. Incremental Gaussian Model-building in Multi-objective EDAs with an Application to Deformable Image Registration. In Proceedings of the 14th Annual Conference on Genetic and Evolutionary Computation (GECCO '12). ACM, New York, NY, USA, 241-248.

[2] P.A.N. Bosman, J. Grahl, and D. Thierens. 2013. Benchmarking Parameter-Free AMaLGaM on Functions With and Without Noise. Evolutionary Computation 21 , 3 (2013), 445-469.

[3] P.A.N. Bosman and D. Thierens. 2003. The Balance Between Proximity and Diversity in Multiobjective Evolutionary Algorithms. IEEE Transactions on Evolutionary Computation 7, 2 (2003), 174-188.

[4] K Deb. 2001. Multi-Objective Optimization Using Evolutionary Algorithms. John Wiley \& Sons, Inc., NY, USA

[5] T.M. Deist and B.L. Gorissen. 2016. High-dose-rate prostate brachytherapy inverse planning on dose-volume criteria by simulated annealing. Physics in Medicine and Biology 61, 3 (2016), 1155.

[6] L. Eapen, C. Kayser, Y. Deshaies, G. Perry, E. Choan, C. Morash, J.E. Cygler, D. Wilkins, and S. Dahrouge. 2004. Correlating the degree of needle trauma during prostate brachytherapy and the development of acute urinary toxicity. International fournal of Radiation Oncolog $y^{*}$ Biolog $y^{*}$ Physics 59, 5 (2004), 13921394.

[7] Peter J. Hoskin, Alessandro Colombo, Ann Henry, Peter Niehoff, Taran Paulsen Hellebust, Frank-Andre Siebert, and Gyorgy Kovacs. 2013. GEC/ESTRO recommendations on high dose rate afterloading brachytherapy for localised prostate cancer: An update. Radiotherapy and Oncology 107, 3 (2013), 325 - 332. DOI http://dx.doi.org/10.1016/j.radonc.2013.05.002

[8] S.K. Kang, R.H. Chou, R.K. Dodge, R.W. Clough, H.L. Kang, M. Bowen, B.A Steffey, S.K. Das, S. Zhou, A.W. Whitehurst, and others. 2001. Acute urinary toxicity following transperineal prostate brachytherapy using a modified Quimby loading method. International fournal of Radiation Oncolog $y^{*}$ Biolog $y^{*}$ Physics 50, 4 (2001), 937-945.

[9] A. Karabis, P. Belotti, and D. Baltas. 2009. Optimization of Catheter Position and Dwell Time in Prostate HDR Brachytherapy using HIPO and Linear Programming. Springer Berlin Heidelberg, Berlin, Heidelberg, 612-615.

[10] H.N. Luong and P.A.N. Bosman. 2012. Elitist Archiving for Multi-Objective Evolutionary Algorithms: To Adapt or Not to Adapt. In International Conference on Parallel Problem Solving from Nature. Springer, 72-81.

[11] Dutch Cancer Registry managed by IKNL (c) January 2017. Prostaatkanker (Prostaatcarcinoom). http://www.prostaat.nl/aandoeningen/ prostaatkanker-prostaatcarcinoom/.

[12] K.L. Sadowski, P.A.N. Bosman, and D. Thierens. 2016. Learning and Exploiting Mixed Variable Dependencies with a Model-Based EA. In Evolutionary Computation (CEC), 2016 IEEE Congress on. IEEE, 4382-4389.

[13] D. Thierens and P.A.N. Bosman. 2011. Optimal Mixing Evolutionary Algorithms. In Proceedings of the Genetic and Evolutionary Computation Conference (GECCO '11). ACM, New York, NY, USA, 617-624.

[14] C. Vargas, M. Ghilezan, M. Hollander, G. Gustafson, H. Korman, J. Gonzalez, and A. Martinez. 2005. A new model using number of needles and androgen deprivation to predict chronic urinary toxicity for high or low dose rate prostate brachytherapy. The fournal of urology 174, 3 (2005), 882-887. (a) Selected patient 1 solution
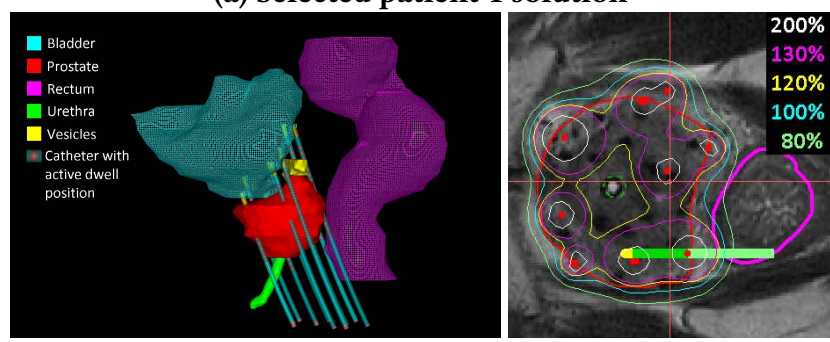

\begin{tabular}{|c|c|}
\hline Prostate & Vesicles \\
\hline$V_{100}=96.39 \%$ & $V_{80}=96.40 \%$ \\
\hline
\end{tabular}

\begin{tabular}{|c|c|c|c|}
\hline Prostate & Bladder & Rectum & Urethra \\
\hline$V_{150}=27.20 \%$ & $V_{86}=0.58 c c$ & $V_{78}=0.78 c c$ & $V_{110}=0.09 c c$ \\
\hline$V_{200}=11.05 \%$ & $V_{74}=1.78 c c$ & $V_{74}=1.09 c c$ & \\
\hline
\end{tabular}

(b) Selected patient 2 solution
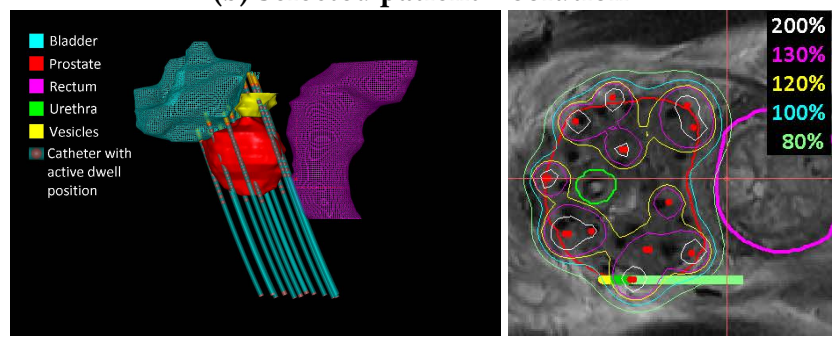

\begin{tabular}{|c|c|c|c|}
\hline Prostate & Vesicles & & \\
\hline$V_{100}=98.53 \%$ & $V_{80}=99.00 \%$ & & \\
\hline Prostate & Bladder & Rectum & Urethra \\
\hline$V_{150}=19.72 \%$ & $V_{86}=0.52 c c$ & $V_{78}=0.81 c c$ & $V_{110}=0.08 c c$ \\
\hline$V_{200}=6.76 \%$ & $V_{74}=1.82 c c$ & $V_{74}=1.21 c c$ & \\
\hline
\end{tabular}

(c) Selected patient 3 solution
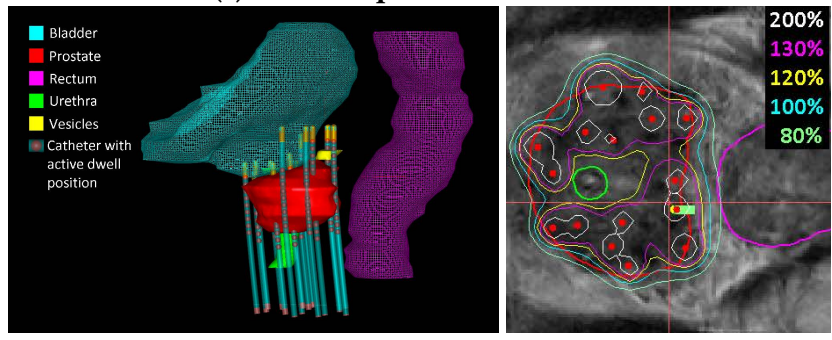

\begin{tabular}{|c|c|}
\hline Prostate & Vesicles \\
\hline$V_{100}=95.29 \%$ & $V_{80}=95.50 \%$ \\
\hline
\end{tabular}

\begin{tabular}{|c|c|c|c|}
\hline Prostate & Bladder & Rectum & Urethra \\
\hline$V_{150}=27.24 \%$ & $V_{86}=0.85 c c$ & $V_{78}=0.50 c c$ & $V_{110}=0.09 c c$ \\
\hline$V_{200}=8.35 \%$ & $V_{74}=1.87 c c$ & $V_{74}=0.69 c c$ & \\
\hline
\end{tabular}

Figure 5: Selected solutions from the Pareto fronts of patients 1,2 and 3 resulting in a high-quality plan with 10,12 and 16 catheters, respectively. The figures on the right show the corresponding isodose lines in selected slices on the corresponding MRI scans, where red dots are active dwell positions. The tables show the corresponding DVH indices. All DVH index values satisfy the clinical constraints. 